\title{
STIMULATED RAMAN-UV OPTICAL DOUBLE RESONANCE SPECTROSCOPY OF VAN DER WAALS COMPLEXES OF BENZENE
}

\author{
TAKAYUKI EBATA, SEIICHI ISHIKAWA, MITSUO ITO ${ }^{1}$ and \\ SHI-AKI HYODO \\ Chemistry Department, Faculty of Science, Tohoku University, Sendai 980, Japan \\ \#Toyota Central Research \& Development Laboratories, Inc. 41-1 \\ Aza Yokomichi, Oaza Nagakute, Nagakute-cho, \\ Aichi-gun, Aichiken 480-11, Japan \\ ${ }^{1}$ Institute for Molecular Science, Myodaiji, Okazaki 444, Japan
}

(Received 3 April 1993)

\begin{abstract}
Spectroscopic and dynamical studies of the van der Waals complexes of benzene-Ar, benzene- $\mathrm{N}_{2}$ and benzene dimer in the electronically ground state $\left(S_{0}\right)$ have been performed by the use of stimulated Raman-UV optical double resonance spectroscopy. The vibrational frequency of mode 1 of the complexes and the decay rate constants of the $1_{1}$ levels $\left(\sim 993 \mathrm{~cm}^{-1}\right)$ have been measured. The vibrational frequency shifts of mode 1 in $S_{0}$ upon complex formation were less than $1 \mathrm{~cm}^{-1}$ for all the complexes. On the other hand, the decay rate constant due to intramolecular vibrational redistribution and/or vibrational predissociation showed a drastic change. The decay rate constant of the 1, level of the benzene dimer was $2.4 \times 10^{7} \mathrm{~s}^{-1}$, while those of the benzene-Ar and benzene- $\mathrm{N}_{2}$ complexes were much smaller than $1 \times 10^{6} \mathrm{~s}^{-1}$. It was also found that the decay rate constant in $S_{0}$ is much smaller than that in $S_{1}$. For the benzene dimer, the depolarization ratio of Raman band of mode 1 was recalculated by taking into account of the anisotropy of the polarizability of benzene molecule. The comparison between the observed and calculated polarization ratio shows that the benzene dimer has the "T-shaped" structure which was proposed by the group of Felker.
\end{abstract}

KEY WORDS: Stimulated Raman, van der Waals complexes

\section{INTRODUCTION}

Spectroscopy and dynamics of van der Waals complexes have been a main subject for the past ten years. Most of the works have been motivated for elucidating many phenomena observed in liquid phase such as local structure and relaxation process on a microscopic level. These complexes are generally produced by supersonic expansion and are studied by sensitive spectroscopic means, such as laser induced fluorescence spectroscopy and resonance enhanced multiphoton ionization spectroscopy. Geometrical structure of the complexes was discussed on the basis of the 
observed spectral red shift of the electronic transition ${ }^{1-15}$ and rotational structure of the vibronic band. ${ }^{16-23}$ The relaxation dynamics have also been studied by dispersed fluorescence ${ }^{24-38}$ and pump-and-probe techniques. ${ }^{39-44}$ Through these studies, our knowledge for the complexes in their first excited states was considerably accumulated.

We are concerned here with van der Waals complexes in the electronically ground state $\left(\mathrm{S}_{0}\right)$. Compared with numerous studies for the complexes in the $\mathrm{S}_{1}$ state, corresponding studies in $S_{0}$ are still limited. There are several reasons for this. The cross sections of the infrared and spontaneous Raman transition traditionally used for $S_{0}$ are much smaller than the electronic transition. Moreover, the vibrational frequency shift upon complex formation is generally so small that it is difficult to discriminate a specific species from the mixture of many kinds of complex in the studies based on usual vibrational spectroscopy. Stimulated Raman-UV optical double resonance spectroscopy or ionization detected stimulated Raman spectroscopy (IDSRS) is one of the powerful techniques to overcome such difficulties. ${ }^{45-47}$ In this spectroscopy, a van der Waals complex is effectively pumped to a specific vibrational level by stimulated Raman process. The vibrationally excited complex is monitored by resonant enhanced multiphoton ionization (REMPI) through an electronically excited state (normally the $\mathrm{S}_{1}$ state) by use of a tunable UV laser. Since the electronic energy is different for each complex and the complex can be separated by the mass of the produced ion, the identification of each complex becomes possible. The decay of the vibrationally excited complex can also be directly measured by changing the delay time between Raman pumping lasers and the probing laser. Recently, Felker and co-workers successfully measured the vibrational spectra of the complexes containing phenol, ${ }^{48}$ benzene, ${ }^{49-53}$ and carbazole ${ }^{54}$ by using this spectroscopy and ionization loss stimulated Raman spectroscopy (ILSRS). Very recently, we also reported the study on benzene and its dimer by using this spectroscopy and showed that the depolarization ratio of a Raman line is very useful in discussing the structure of the complex..$^{55}$ It was found that the depolarization ratio of the totally symmetric Raman line of benzene drastically changes by the dimer formation.

In the present work, we have studied several van der Waals complexes of benzene including, benzene dimer, benzene-Ar and benzene- $\mathrm{N}_{2}$ complexes by stimulated Raman-UV optical double resonance spectroscopy. There are two main subjects in the present study. First is the structure of the benzene dimer. In our previous work, we concluded that the two Raman mode 1 bands showing the different depolarization ratios and different vibrational frequencies are assigned to the dimers having different structures, that is "T-shaped" and "sandwich" dimers, by evaluating the depolarization ratios of the dimer based on the local field model, ${ }^{56}$ where we assumed an isotropic polarizability for the benzene molecule. On the other hand, Felker et al. proposed that the two bands are assigned to individual benzene molecules of " $\mathrm{T}$ shaped" dimer by measuring the Raman and electronic spectra of the isotope substituted benzene dimers (isotopomers). ${ }^{53}$ So, we reevaluated here the depolarization ratio of the dimer by taking account of the anisotropy of the polarizability of benzene, which was not included in the previous analysis. It was found that the observed two depolarization ratios correspond to those of benzene molecules on 
different site of the "T-shaped" dimer. On the other hand, the calculated ratio of the sandwich dimer did not agree with the observed one.

Secondly, the decay rate constants of the benzene complexes after exciting their $1_{1}$ levels in $S_{0}$ have been measured. In contrast with many studies on the dynamics of the vibrationally excited complex in $S_{1}$, corresponding study in $S_{0}$ is still very few. The dispersion and exchange forces which determines the intermolecular binding energy of a complex depend on its electronic structure. Therefore, the change in the electronic state will naturally affect the dynamics of the complex. However, it is not well known how does the change in the electronic state affect the vibrational relaxation processes such as intramolecular vibrational redistribution (IVR) and vibrational predissociation (VP). The IVR/VP processes of the van der Waals complexes of benzene have been studied for the $S_{1}$ state and their decay rate constants have been obtained. The purpose of this work is to answer for the question "how the vibrational relaxation rate constant in $S_{1}$ is different from that in $S_{0}$ "'.

\section{EXPERIMENTAL}

Figure 1 shows the experimental setup and the excitation scheme of stimulated Raman-UV optical double resonance spectroscopy for jet-cooled molecules. The van der Waals complex of benzene formed in a supersonic free jet was first pumped to a specific vibrational level ( $1_{1}$ level in the present case) by stimulated Raman pumping with $v_{1}$ and $v_{2}$. After certain delay time, the vibrationally excited complex was ionized by $(1+1)$ resonant enhanced multiphoton ionization (REMPI) through the $\mathrm{S}_{1}$ state by using third laser beam $\left(v_{3}\right)$.
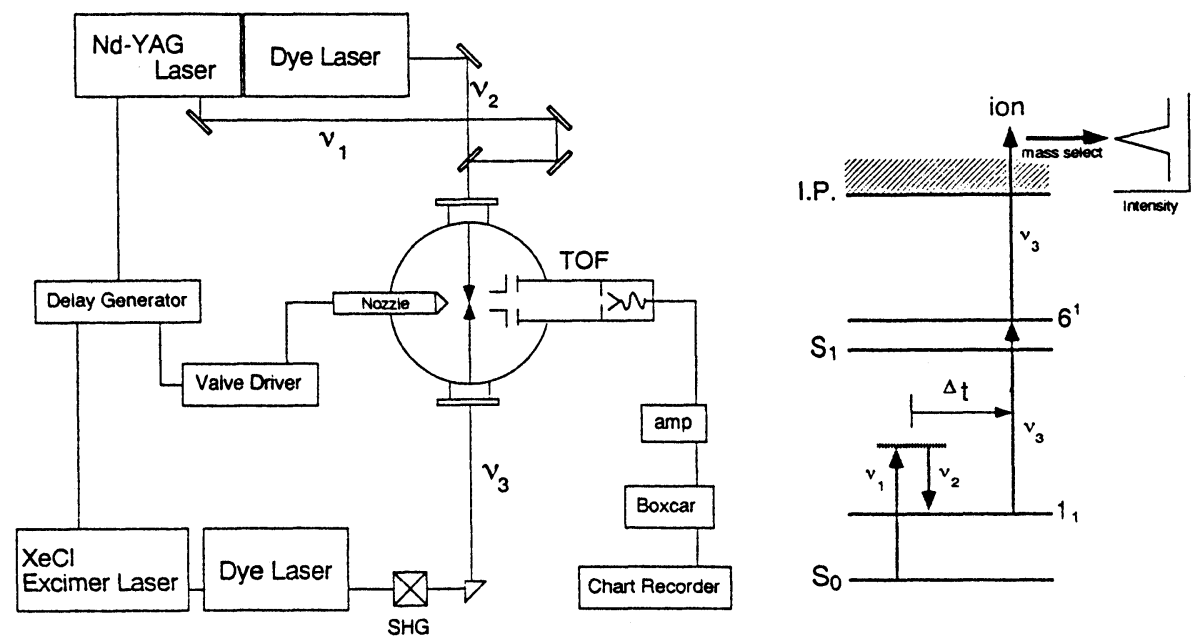

Figure 1 Experimental setup and excitation scheme of stimulated Raman-UV optical double resonance spectroscopy of jet cooled molecular complexes. 
$v_{1}$ and $v_{2}$ were the second harmonic $(532 \mathrm{mn})$ of a Nd:YAG laser (Quantel YG 581-10) and the output of a Nd:YAG pumped dye laser (Quantel TDL 50), respectively. Typical laser powers of $v_{1}$ and $v_{2}$ were $30 \mathrm{~mJ}$ and $20 \mathrm{~mJ}$, respectively, and the two laser beams were combined by a beam combiner and then introduced coaxially into a vacuum chamber. A half of the laser power is lost at the beam combiner and actual laser intensity used for Raman pumping was $\sim 10 \mathrm{~mJ}$. For the measurement of the depolarization ratios, the polarization direction of $v_{2}$ was fixed and that of $v_{1}$ was changed by a Babinet Soleile's compensator. Since the reflection coefficient of the beam combiner is different for s- and p-waves, a neutral density filter was placed to adjust $v_{1}$ laser power. The degree of linear polarization of $v_{2}$ laser beam was $1: 400$. The laser beams were focused $15 \mathrm{~mm}$ downstream of the nozzle by an $f=250 \mathrm{~mm}$ lens. The signal intensify was proportional to the pumping laser powers and the spectral broadening was not observed under the present experimental condition. The third laser beam, $v_{3}$, was counterpropagated to the Raman pumping beams. The $v_{3}$ laser beam was the second harmonic of an excimer laser pumped dye laser (Lambda Physik LPX100/FL2002) and its power was a few tens of $\mu \mathrm{J}$. The beam was either unfocused or slightly focused by an $\mathrm{f}=1 \mathrm{~m}$ lens. The linewidth of $v_{2}$ laser was $0.3 \mathrm{~cm}^{-1}$ and that of $v_{3}$ laser was $0.5 \mathrm{~cm}^{-1}$. Their frequencies were determined by a calibrated $0.75 \mathrm{~m}$ double-monochromator with the accuracy of $\pm 0.5 \mathrm{~cm}^{-1}$. The delay time between Raman pumping lasers and the probe laser was changed by a digital delay generator. The time jitter between the two lasers was $\pm 3 \mathrm{~ns}$. Because our ND:YAG laser system is operated under multi-axial mode condition, the $532 \mathrm{~nm}$ laser light $\left(v_{1}\right)$ consists of several lines within the $\sim 1.5 \mathrm{~cm}^{-1}$ full width. Therefore, the fine structure of the Raman band observed in the stimulated Raman spectrum is due to this multi-mode operation and is nothing to do with the rotational structure.

The benzene pressure was controlled by a thermoregulator. The temperature was cooled to $-10 \mathrm{C}$ to prevent from the formation of higher clusters. The gaseous mixture of $\mathrm{Ar} / \mathrm{He}$ or $\mathrm{N}_{2} / \mathrm{He}$ was expanded into vacuum through a $400 \mu \mathrm{m}$ pulsed nozzle. Typical mixing ratio of $\mathrm{Ar}$ or $\mathrm{N}_{2}$ to $\mathrm{He}$ was $20 \%$. The nozzle and lasers were operated at $10 \mathrm{~Hz}$. Figure 2 shows two types of mass selection system used in the present experiment. In Figure 2(a), the laser beams are focused at the position of $10 \mathrm{~mm}$ upstream of the extracting position. Since velocities of the benzene monomer and dimer coexisting in the jet are not so different, the mass-selection can be simply done by adjusting the voltage of the repeller to lead the specific ion to the entrance of the detector chamber. The ions travel for $10 \mathrm{~cm}$ to reach the detector after passing through the entrance of the detection chamber. In Figure 2(b) is shown the Wiley Mclaren TOF mass spectrometer. A slight modification was done for the repeller and extractor to prevent from interfering the free jet expansion. The flight length was $25 \mathrm{~cm}$ and typical voltages of the repeller, extractor and drift tube were $300 \mathrm{~V}$, $15 \mathrm{~V}$ and $-250 \mathrm{~V}$, respectively. Figure 2(c) shows the time-of-flight spectrum measured at $20 \mathrm{~ns}$ after Raman pumping to mode 1 of benzene dimer. The ions were detected by an electron multiplier (Murata Ceratron) and the signal from the detector was amplified by an amplifier (NF Model BX-31) and processed by a boxcar integrator. Benzene was purchased from Wako Chemicals and was degassed by pump-thaw-freeze cycle. 
(a)

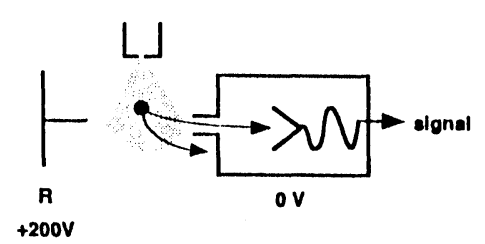

(b)

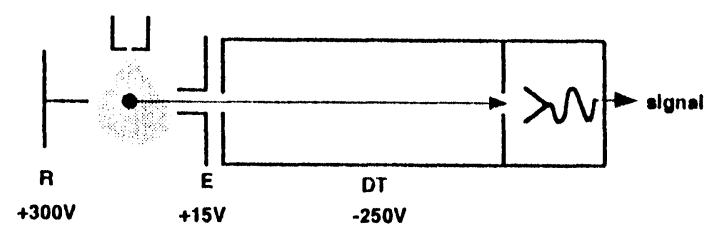

(c)

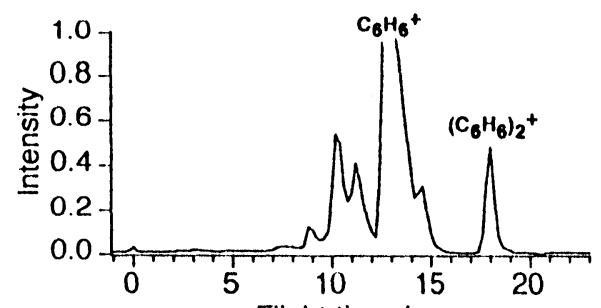

Flight time / $\mu \mathrm{s}$

Figure 2 (a) and (b) Setups for mass selection used and (c) TOF spectrum measured by using the setup (b).

\section{RESULTS AND DISCUSSION}

\section{Vibrational spectroscopy of benzene complexes}

\section{A. Benzene dimer}

Figure 3(a) shows the mass-selected $(1+1)$ REMPI spectrum of the $66_{0}{ }^{1} 1_{1}{ }^{0}$ region of benzene dimer measured at $20 \mathrm{~ns}$ after exciting the benzene dimer to the $1_{1}$ level. Observed red shift from the $6_{0}{ }^{1} 1_{1}{ }^{0}$ band of the benzene monomer was $44 \mathrm{~cm}^{-1}$, which is very close to the red shift for the $6_{0}{ }^{1}$ band. As was explained previously, the spectrum of benzene dimer consists of two types of band..$^{55}$ One shows sharp and doublet peaks observed at 37,567 and $37,570.5 \mathrm{~cm}^{-1}$ and the other is the weaker bands showing a progression of low frequency $\left(\sim 17 \mathrm{~cm}^{-1}\right)$ van der Waals mode with the strongest band at $37,591 \mathrm{~cm}^{-1}$. Figure 4 shows the stimulated Raman spectra of 
(a) Benzene dimer

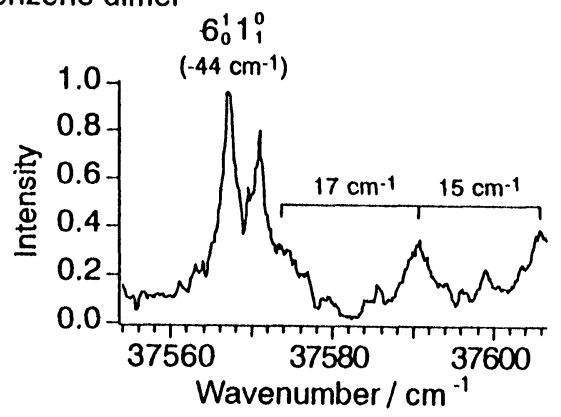

(b) Benzene - Ar

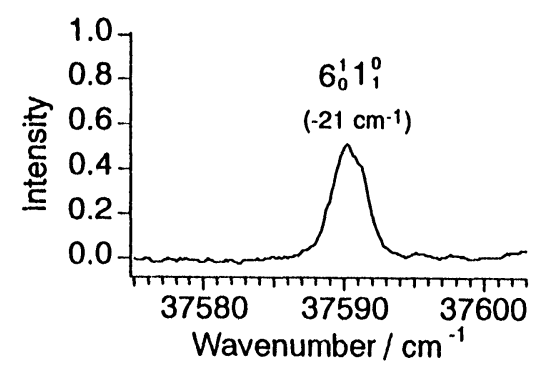

(c) Benzene $-\mathrm{N}_{2}$

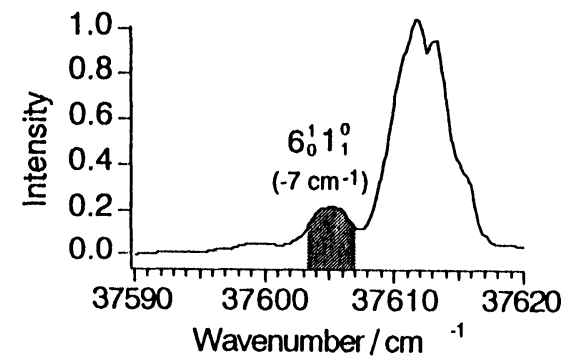

Figure 3 Stimulated Raman-UV optical double resonance spectra of (a) benzene dimer, (b) benzene-Ar $(1: 1)$ complex and (c) benzene- $\mathrm{N}_{2}(1: 1)$ complex showing the $6_{0}{ }^{1} 1_{1}{ }^{0}$ band.

mode 1 of benzene dimer measured (b) when $v_{3}$ is fixed on $37,591 \mathrm{~cm}^{-1}$ and (c) when $v_{3}$ is fixed on $37,567 \mathrm{~cm}^{-1}$. Figure 4(a) shows the stimulated Raman spectrum of the mode 1 of the benzene monomer for comparison. These spectra were obtained by scanning $v_{2}$ while $v_{3}$ is fixed to the respective $6_{0}{ }^{1} 1_{1}{ }^{0}$ bands. As was mentioned in the experimental section, the appearance of several peaks is due to the multi-mode operation of Nd:YAG laser which is used as $v_{1}$ and is nothing to do with the rotational structure. Though the appearance of the Raman spectrum is complicated due to the multi-mode operation, the vibrational frequency shift of mode 1 upon the complex formation can be determined with a high accuracy of $\pm 0.3 \mathrm{~cm}^{-1}$. The frequency change of mode 1 by the dimer formation is $-0.6 \mathrm{~cm}^{-1}$ when $v_{3}$ is fixed on 
(a) Benzene monomer

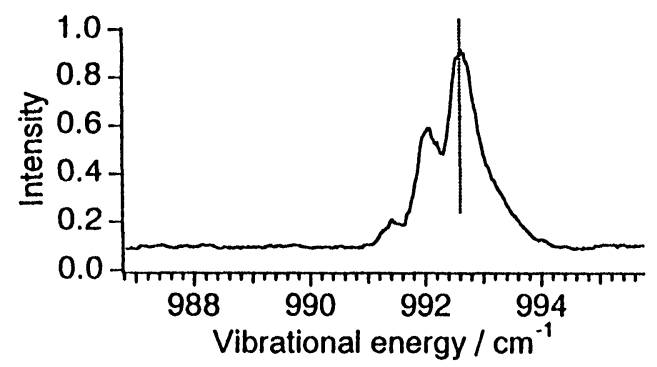

(b) Benzene dimer

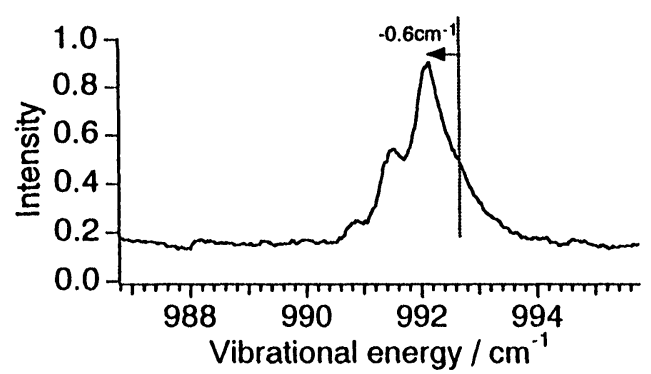

(c) Benzene dimer

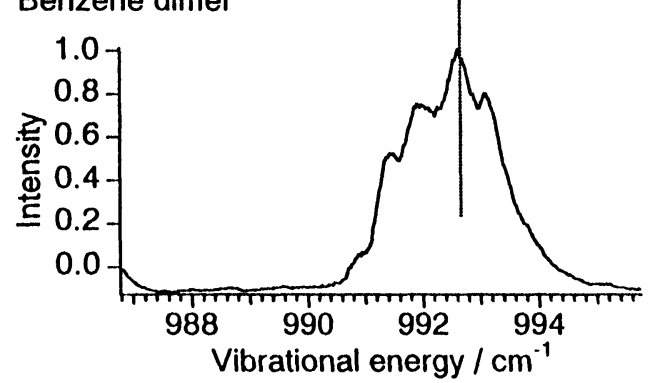

Figure 4 Stimulated Raman spectra of mode 1 of benzene monomer (a), and benzene dimer (b), (c) : (b) $v_{3}$ is fixed on the band at $37,591 \mathrm{~cm}^{-1}$ and (c) $v_{3}$ is fixed on the band at $37,567 \mathrm{~cm}^{-1}$.

the band at $37,591 \mathrm{~cm}^{-1}$. On the other hand, no frequency change was observed when $v_{3}$ is fixed on the band at $37,567 \mathrm{~cm}^{-1}$. Therefore, we have two Raman bands of mode 1, whose Raman frequencies differ by $0.6 \mathrm{~cm}^{-1}$ and the electronic spectra associated with these Raman bands are also quite different. As was reported previously, the depolarization ratios for these two bands were found to be also greatly different. While the depolarization ratio for benzene monomer was 0.008 , that of the band of Figure 4(b) was 0.02 and that of the band of Figure 4(c) was 0.1 .

In the previous paper, the two bands were assigned to belong to the different isomers of benzene dimer, that is (a) "T-shaped" dimer and (b) "sandwich" dimer, which was determined by the comparison of the measured depolarization ratios with the 
calculated ones. ${ }^{55}$ On the other hand, the group of Felker reported that the two bands belong to the transitions from the different site of benzenes in the T-shaped dimer from the analysis of the spectra of the isotope substituted benzene dimers..$^{53}$ Very recently, Scherzer et al. also showed the evidence of the T-shaped dimer by hole-burning spectroscopy ${ }^{57}$ Therefore, there is a discrepancy in the interpretation of the observed spectra. Our previous assignment was based on the calculation of the local field effect on the depolarization ratio by assuming isotropic polarizability for the benzene molecules. As a result, two different intermolecular distances were obtained for the observed different depolarization ratios. However, the polarizability of benzene is not isotropic and we also measured the non zero depolarization ratio of mode 1 of benzene monomer. Therefore, the assumption of the isotropic polarizability of benzene molecule is not adequate to describe the structure of the dimer.

(a) T-shaped structure

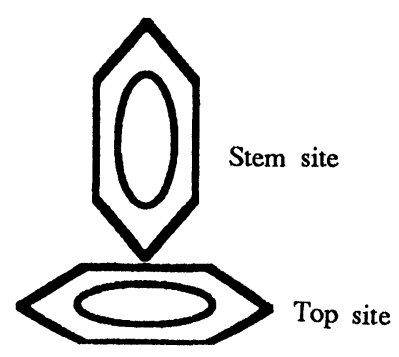

(b) Sandwich structure

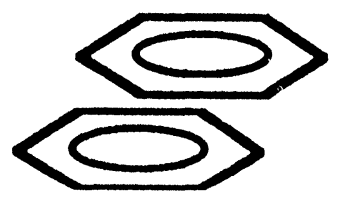

In the present study, the calculation of the Raman depolarization ratio of mode 1 of the dimer has been done for the above two dimer geometries, by taking account of the anisotropy of benzene molecule. As was reported in the previous paper, the local field was described as the effect of induced polarization on each benzene molecule produced by an electric field of incident light. ${ }^{56} \mathrm{Here}$, we assumed that the polarizability of a benzene molecule is unchanged in dimer conformation, which is acceptable when deformation of the electronic structure of the molecule is negligibly small upon the dimer formation. We also assumed that the vibrations are localized on each site of the dimer. In the actual dimer, the two mode 1 vibrations of each benzene molecules will be mixed to form a normal mode of the dimer. However the degree of the mixing of the two vibrations in the dimer can not be determined at present and the detailed intermolecular potential which is necessary to evaluate the mixing has not been reported yet. Up to now, several theoretical calculations have been done in terms of molecular orbital theory to investigate the detailed intermolecular potential and also there have been many discussions on the stable structure of the benzene dimer. ${ }^{58-62}$ However, a detailed analysis like energy decomposition analysis ${ }^{63}$ or that of vibrational structure has not been reported enough to discuss the coupling of molecular vibrations. So, the present analysis has been performed by assuming that the vibrations are localized on each site of benzene molecules. 
First, we will introduce three types of coordinate system as are shown in Figure 5. An incident light irradiates a dimer along the positive direction of $X$-axis and the electric field is polarized in Z-axis of the laboratory fixed coordinate. The matrix of transforming the dimer fixed coordinate to the laboratory fixed coordinate is denoted as $\Phi$ and that of transforming the molecular fixed coordinate to the dimer fixed coordinate as $\Phi_{\mathrm{a}}\left(\right.$ or $\left.\Phi_{\mathrm{b}}\right)$. Here the subscript denotes benzene molecule in the different site.

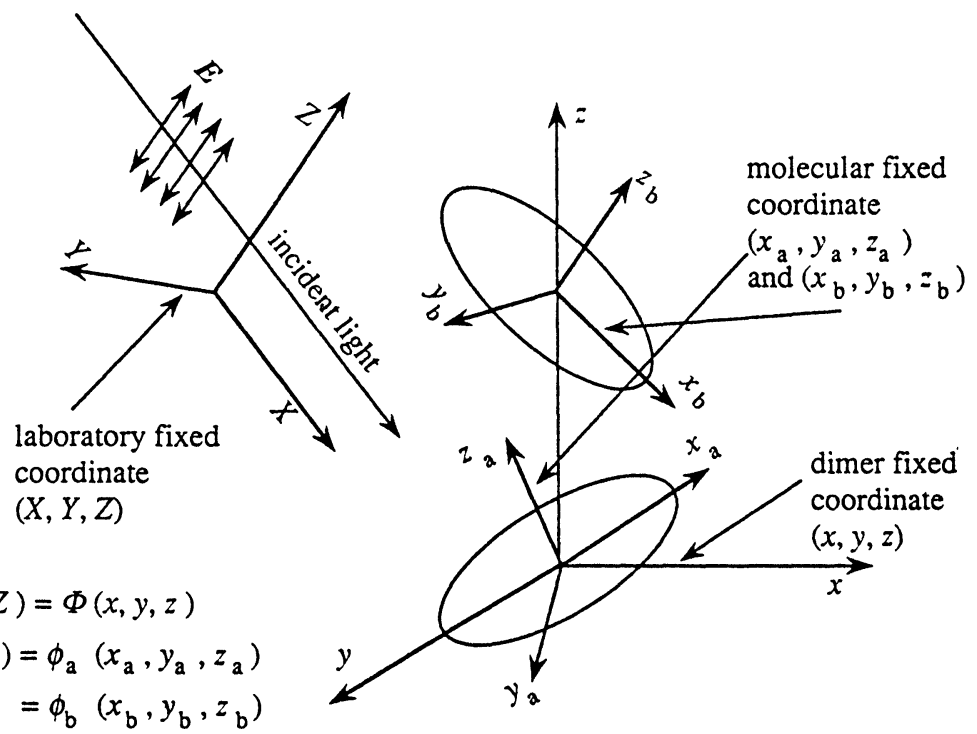

Figure 5 Three coordinates used in the calculation: the laboratory fixed coordinate, the molecular fixed coordinate and the dimer fixed coordinate.

The electric field of incident light in the laboratory fixed coordinate,

$$
\boldsymbol{E}=\left(0,0, \mathrm{E}_{\mathrm{z}}\right)
$$

is transformed as $\Phi \boldsymbol{E}$ in the dimer fixed coordinate and $\widetilde{\Phi}_{\mathrm{a}(\mathrm{b})} \boldsymbol{\Phi} \boldsymbol{E}$ in the molecular fixed coordinate, where $\sim$ denotes inverse matrix. The polarizability of benzene molecules, $\boldsymbol{\alpha}$, is written as

$$
\boldsymbol{\alpha}=\left(\begin{array}{ccc}
\alpha_{\perp} & 0 & 0 \\
0 & \alpha_{\perp} & 0 \\
0 & 0 & \alpha_{/ /}
\end{array}\right)
$$

in a molecular fixed coordinate. The induced dipole moment on each benzene molecule generated by external field, $\widetilde{\Phi}_{\mathrm{a}(\mathrm{b})} \Phi \boldsymbol{E}$, is expressed as

$$
\boldsymbol{\mu}_{\mathrm{a}(\mathrm{b})}=\boldsymbol{\alpha} \widetilde{\Phi}_{\mathrm{a}(\mathrm{b})} \Phi \boldsymbol{E},
$$

in a molecular fixed coordinate and it is transformed to 


$$
\boldsymbol{\mu}_{\mathrm{a}(\mathrm{b})}=\Phi_{\mathrm{a}(\mathrm{b})} \boldsymbol{\alpha} \widetilde{\Phi}_{\mathrm{a}(\mathrm{b})} \Phi \boldsymbol{E},
$$

in the dimer fixed coordinate. The external field oscillates with the incident light frequency, $\omega / 2 \pi$, and the induced moment generates electromagnetic wave around each molecule, which gives the additional field on the counterpart molecule in the dimer. The induced dipole on benzene on site (b) produces an electric field as,

$$
\mathbf{F}_{\mathrm{a} \leftarrow \mathrm{b}}^{\mathrm{D}}=\frac{\boldsymbol{\mu}_{\mathrm{b}}^{\mathrm{D}}}{\mathbf{R}_{\mathrm{ab}}^{3}}-\frac{3\left(\boldsymbol{\mu}_{\mathrm{b}}^{\mathrm{D}} \cdot \mathbf{R}_{\mathrm{ab}}\right) \mathbf{R}_{\mathrm{ab}}}{\mathbf{R}_{\mathrm{ab}}^{5}},
$$

where $\mathbf{R}_{\mathrm{ab}}$ is the vector denoting the distance between the center of two benzene molecules and $\mathbf{R}_{\mathrm{ab}}=\left|\mathbf{R}_{\mathrm{ab}}\right|$. Therefore, the electric field that the benzene on site (a) feels is expressed as

$$
\mathrm{f}_{\mathrm{a}}=\widetilde{\Phi}_{\mathrm{a}}\left(\Phi \mathrm{E}+\mathbf{F}_{\mathrm{a} \leftarrow \mathrm{b}}^{\mathrm{D}}\right)
$$

By the same way the electric field that the benzene on site (b) feels can be written by exchanging the subscripts $a$ and $b$ in this equation. The induced moment described as the product of this local electric field and Raman scattering tensor, $\boldsymbol{\alpha}^{\prime}$, gives a Raman scattering.

The Raman scattering tensor is defined by a polarizability tensor derivative with respect to a particular normal coordinate of intramolecular vibrational mode, $\mathrm{Q}$,

$$
\boldsymbol{\alpha}_{\mathrm{Q}}^{\prime} \equiv\left(\frac{\partial \boldsymbol{\alpha}}{\partial \mathrm{Q}}\right)_{0}
$$

Since only mode 1 of benzene is considered here, we omit the subscript $\mathrm{Q}$ in $\alpha^{\prime}$. The induced moment with a Raman frequency, $\left(\omega \pm \omega_{\mathrm{Q}}\right) / 2 \pi$, can be written by

$$
\mathbf{M}_{\mathrm{a}(\mathrm{b})=}=\Phi \Phi_{\mathrm{a}(\mathrm{b})} \boldsymbol{\alpha}^{\prime} \tilde{\Phi}_{\mathrm{a}(\mathrm{b})}\left(\Phi \mathrm{E}+2 \mathbf{F}_{\mathrm{a} \leftarrow \mathrm{b}(\mathrm{b} \leftarrow \mathrm{a})}^{\mathrm{D}}\right),
$$

in the laboratory fixed coordinate. The factor, 2 , in the second term of this equation is due to the following reason. It is found that by replacing $\boldsymbol{\alpha}$ by $\boldsymbol{\alpha}^{\prime}$ in eq. (3), the electric field described in eq. (5) has a frequency of $\left(\omega \pm \omega_{Q}\right) / 2 \pi$, which is equal to the Raman frequency. The induced moment obtained by operating a polarizability tensor, $\boldsymbol{\alpha}$, to this field gives Raman scattering with the same frequency. The expression of this induced moment is unchanged by the above replacement. The effect of local field expressed by eq. (5), therefore, appears as a factor of 2 in the final description of induced moment of eq. (8). The intensity of a component of polarized Raman scattering is then expressed by

$$
\mathrm{I}_{\mathrm{G}} \propto\left\langle\left\{\Phi \Phi_{\mathrm{a}(\mathrm{b})} \boldsymbol{\alpha}^{\prime} \tilde{\Phi}_{\mathrm{a}(\mathrm{b})}\left(\Phi \mathbf{E}+2 \mathbf{F}_{\mathrm{a} \leftarrow \mathrm{b}(\mathrm{b} \leftarrow \mathrm{a})}^{\mathrm{D}}\right)\right\}_{\mathrm{G}}^{2}\right\rangle,
$$

where $\mathrm{G}$ corresponds to the component of the laboratory fixed coordinate (X, $\mathrm{Y}$ or $\mathrm{Z})$ and \langle\rangle means operation of the orientational average. The depolarization ratio $\rho$ can be determined by

$$
\rho=\mathrm{I}_{\mathrm{G}} / \mathrm{I}_{\mathrm{Z}}(\mathrm{G}=\mathrm{X} \text { or } \mathrm{Y})
$$


in the present coordinate system. The explicit expression of $\mathrm{I}_{\mathrm{G}}$ or $\rho$ is defined by the geometry of the dimer. In eq. (9), the geometrical factor is introduced by the transformation matrix, $\widetilde{\Phi}_{\mathrm{a}(\mathrm{b})}$, from the relative orientation and local field, $\mathbf{F}_{\mathrm{a} \leftarrow \mathrm{b}(\mathrm{b} \leftarrow \mathrm{a})}^{\mathrm{D}}$.

The transformation matrix between the two coordinate was described in terms of Eulerian angle. ${ }^{64}$ The expressions of depolarization ratio corresponding to the two dimer geometry, (a) T-shape and (b) sandwich, were obtained by substituting particular values to Eulerian angles. The resultant expressions of the Raman scattering intensities for eách polarized component are shown in Table 1. A general expression of the scattering intensity can not be practically derived for any dimer structure because of considerably large amount of terms included in this expression. The results in Table 1 are ones for particular structures of dimers with anisotropic polarizability. To derive those expressions, a computer program Mathematica ${ }^{\mathrm{TM}}$ (enhanced ver. 1.2.1 f33) was operated on Macintosh IIci with $8 \mathrm{MB}$ memory.

By substituting the values of polarizability tensor, the Raman scattering tensor, the possible intermolecular distances for each molecular configurations to the expressions in Table 1 and the depolarization ratios for two dimer structures were obtained. The parameters used in the numerical estimation and the calculated depolarization ratios are shown in Tables 2 and 3, respectively. As can be seen in Table 3, the "sandwich" structure gives much larger depolarization ratio than the observed ones. On the other hand, the calculated depolarization ratios of the " $T$ shape" structure well reproduced the observed ones. The benzene on top site gives the depolarization ratio $\rho=0.072 \sim 0.1$ and the benzene on stem site gives $\rho=0.011 \sim 0.017$, which agree with the previously observed ratios of 0.1 and 0.02 , respectively. Therefore the present analysis essentially supports the T-shaped dimer structure proposed by the group of Felker. However, our interpretation about which of the observed vibrational band corresponds to which site of benzene is different from theirs. We assigned that the mode 1 vibration whose frequency is $0.6 \mathrm{~cm}^{-1}$ lower than the monomer and $\rho=0.02$ corresponds to the benzene on stem site and the vibration whose frequency was almost equal to that of the monomer and $\rho=0.1$ corresponds to that on the top site, which is opposite to the assignment by the group of Felker. The mode 1 is the ring breathing in-plane vibration and for the top site of benzene its coordinate is perpendicular to the benzene on stem site. Therefore, if the benzene on stem site dose not affect the $\pi$ electron of the benzene of top site, the coupling of mode 1 of top site benzene with the motion of stem site of benzene is weak and therefore vibrational frequency change will be small. On the other hand, the mode 1 vibration of benzene on the stem side will be coupled with the motion of top site of benzene, which will result in the vibrational red shift for the mode 1 vibration as was observed experimentally. Therefore, from the analysis of the depolarization ratios and the vibrational frequency shift, we conclude that the mode 1 vibration whose frequency is shifted to red by $0.6 \mathrm{~cm}^{-1}$ from the monomer in the Raman spectrum corresponds to the vibration of the benzene on the stem site and the vibration whose frequency change was small corresponds to the benzene on top site. 
Table 1 Resulted Analytical Expression of Polarized Raman Intensities for Each Polarization Components in "sandwich" and "T-shape" model structures, $\left(\theta \equiv \alpha_{\perp}^{\prime} / \alpha^{\prime}\right)$.

"sandwich" structure model

perpendicular component ( $X$ or $Y$-polarized in Figure 5)

$$
\frac{\alpha^{\prime 2}}{15}\left(\theta-1+2 \frac{\alpha_{/ /}+\alpha_{\perp} \theta}{\mathrm{R}_{\mathrm{ab}}^{3}}\right)^{2} \mathrm{E}_{\mathrm{Z}}^{2}
$$

parallel component (Z-polarized in Figure 5)

$$
\frac{\alpha^{\prime 2}}{15}\left\{3 \theta^{2}-16 \theta+28+\frac{4}{\mathrm{R}_{\mathrm{ab}}^{3}}(3 \theta-8)\left(2 \alpha_{/ /}+\alpha_{\perp} \theta\right)+\frac{12}{\mathrm{R}_{\mathrm{ab}}^{6}}\left(2 \alpha_{/ /}+\alpha_{\perp} \theta\right)^{2}\right\} \mathrm{E}_{\mathrm{Z}}^{2}
$$

top site benzene of "T-shape" structure model

perpendicular component ( $X$ or $Y$-polarized in Figure 5)

$$
\begin{gathered}
\frac{\alpha_{/ /}^{\prime 2}}{15}\left[(\theta-1)^{2}+\frac{1}{\mathrm{R}_{\mathrm{ab}}^{3}}(\theta-1)\left(5 \alpha_{\perp}+5 \alpha_{/ /} \theta+2 \alpha_{\perp} \theta\right)\right. \\
{\left[+\frac{1}{\mathrm{R}_{\mathrm{ab}}^{6}}\left\{7\left(\alpha_{\perp}+\alpha_{/ /} \theta\right)^{2}+2 \alpha_{\perp} \theta\left(\alpha_{\perp}+\alpha_{/ /} \theta+2 \alpha_{\perp} \theta\right)\right\}\right] \mathrm{E}_{\mathrm{Z}}^{2}}
\end{gathered}
$$

parallel component (Z-polarized in Figure 5)

$$
\begin{gathered}
\frac{\alpha_{/ /}^{\prime 2}}{15}\left[3 \theta^{2}-16 \theta+28+\frac{1}{\mathrm{R}_{\mathrm{ab}}^{3}}\left\{10(\theta-2)\left(\alpha_{\perp}+\alpha_{/ /} \theta\right)-4 \alpha_{\perp}(\theta-6)\right\}\right. \\
{\left[+\frac{1}{\mathrm{R}_{\mathrm{ab}}^{6}}\left\{11\left(\alpha_{\perp}+\alpha_{/ /} \theta\right)^{2}+4 \alpha_{\perp} \theta\left(3 \alpha_{\perp} \theta-\alpha_{/ /} \theta-\alpha_{\perp}\right)\right\}\right] \mathrm{E}_{\mathrm{Z}}^{2}}
\end{gathered}
$$

stem site benzene of " $T$-shape" structure model

perpendicular component (X or Y-polarized in Figure 5)

$$
\begin{gathered}
\frac{\alpha_{/ /}^{\prime 2}}{15}\left[(\theta-1)^{2}+\frac{2}{\mathrm{R}_{\mathrm{ab}}^{3}}(\theta-1)\left(-2 \alpha_{\perp}-2 \alpha_{/ /} \theta+\alpha_{\perp} \theta\right)\right. \\
{\left[+\frac{1}{\mathrm{R}_{\mathrm{ab}}^{6}}\left\{7\left(\alpha_{\perp}+\alpha_{/ /} \theta\right)^{2}+2 \alpha_{\perp} \theta\left(\alpha_{\perp}+\alpha_{/ /} \theta+2 \alpha_{\perp} \theta\right)\right\}\right] \mathrm{E}_{\mathrm{Z}}^{2}}
\end{gathered}
$$

parallel component (Z-polarized in Figure 5)

$$
\begin{gathered}
\frac{\alpha_{/ 2}^{\prime 2}}{15}\left[8 \theta^{2}+4 \theta+3-\frac{2}{\mathrm{R}_{\mathrm{ab}}^{3}}\left\{(4 \theta-1)\left(\alpha_{\perp}+\alpha_{/ /} \theta\right)+2\left(\alpha_{/ /}-4 \alpha_{\perp}\right) \theta^{2}\right\}\right. \\
{\left[+\frac{1}{\mathrm{R}_{\mathrm{ab}}^{6}}\left\{11\left(\alpha_{\perp}+\alpha_{/ /} \theta\right)^{2}+4 \alpha_{\perp} \theta\left(3 \alpha_{\perp} \theta-\alpha_{/ /} \theta-\alpha_{\perp}\right)\right\}\right]}
\end{gathered}
$$

It should be noted that though we discussed only the two possible structures of the dimer, it is also possible to examine other conformation of the dimer. For example, if the two benzene planes are inclined by 30 degree simultaneously from the parallel sandwich structure, the depolarization ratio of $0.06 \sim 0.03$ is obtained with the intermolecular distance of $3.9 \sim 4.5 \AA$, which is smaller by one order of magnitude. 
Table 2 Parameters for the Estimation of Depolarization Ratio of Benzene Dimer.

tensor elements of anisotropic polarizabilitya)

$\alpha_{\| \prime}$ (parallel to molecular axis)

$\alpha_{\perp}$ (perpendicular to molecular axis)

$12.31 \AA^{3}$

ratio of Raman scattering tensor elements ${ }^{\text {b) }}$

$\alpha^{\prime}{ }_{11}: \alpha^{\prime} \mathcal{V} \perp$ (parallel vs. perpendicular)

intramolecular carbon-carbon distance ${ }^{\text {c) }}$

intramolecular carbon-hydrogen distance ${ }^{\text {c) }}$

$: 1.4 \AA$

$: 1.1 \AA$

van der Waals radius of carbon atom ${ }^{\text {c) }}$

$: 1.7 \AA$

van der Waals radius of hydrogen atom ${ }^{\text {c) }}$

$: 1.2 \AA$

a) reference 71

b) obtained from observed depolarization ratio of benzene monomer. reference 55

c) reference 72

Table 3 Estimated Depolarization Ratio of Monomer Totally Symmetric Vibration in Model Structure of Benzene Dimer.

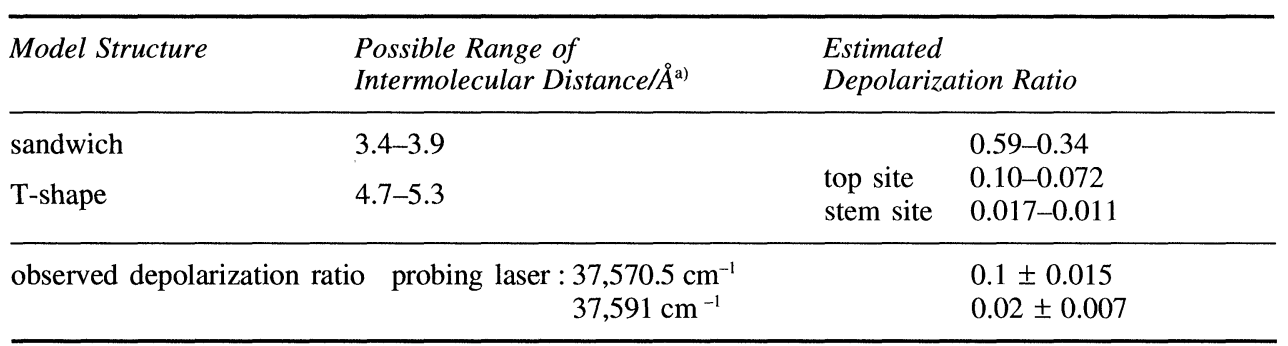

a) Possible intermolecular distances were estimated from molecular structure and van der Waals radius of monomer benzene listed in table 2 and hypothetical intermolecular potential functions obtained from X-ray diffraction study (Reference 73) and semiempirical molecular orbital calculation (Reference 74)

\section{B. Benzene-Ar $(1: 1)$ and benzene- $N_{2}(1: 1)$ complexes}

Figure 3 also shows the $1+1$ REMPI spectrum of (b) benzene-Ar (1:1) and (c) benzene- $\mathrm{N}_{2}(1: 1)$ complexes measured after stimulated Raman pumping to their $1_{1}$ levels. The spectra show the region of $1_{1}{ }^{1} 6_{0}{ }^{1}$ band. Though the mass resolution of TOF mass spectrometer used in the present experiment was not good enough to discriminate benzene- $\mathrm{N}_{2}$ from benzene monomer in the TOF spectrum, the peak of $1_{1}{ }^{0} 6_{0}{ }^{1}$ band of benzene- $\mathrm{N}_{2}$ was observed to be clearly separated from that of benzene monomer as can be seen in the figure. The spectral red shift was $-21 \mathrm{~cm}^{-1}$ for benzene-Ar complex and $-7 \mathrm{~cm}^{-1}$ for benzene- $\mathrm{N}_{2}$ complex, which are close to the red shifts for the $6_{0}{ }^{1}$ band of each complexes. The change in the vibrational frequency of mode 1 was not observed for benzene-Ar complex and was $-0.3 \mathrm{~cm}^{-1}$ for benzene- $\mathrm{N}_{2}$ complex. The frequency change was obtained from the observed stimulated Raman spectrum and also by the comparison of the spectral red shift of the $1_{1}{ }^{0} 6_{0}{ }^{1}$ and $6_{0}{ }^{1}$ bands. As was described previously, the accuracy of the spectra is $\pm 0.3 \mathrm{~cm}^{-1}$. Therefore, for all the van der Waals complexes studied here the vibrational frequency shift upon the complex formation is less than $1 \mathrm{~cm}^{-1}$, indicating a very 
small perturbation on the vibrational potential of mode 1 by the complex formation. As to the benzene-Ar complexes, the group of Felker reported accurate frequency shift of mode 1 for various cluster sizes.

\section{Relaxation of the vibrationally excited benzene complexes}

Figure 6 shows the time profiles of (a) benzene dimer, (b) benzene-Ar, and (c) benzene- $\mathrm{N}_{2}$ complexes measured after pumping to their $1_{1}$ levels. $v_{3}$ is fixed on the $1_{1}{ }^{0} 6_{0}{ }^{1}$ band. For benzene dimer, the same decay curve was obtained both when $v_{3}$ is fixed on the band at $37,567 \mathrm{~cm}^{-1}$ and on the band at $37,591 \mathrm{~cm}^{-1}$. As can be seen in Figure $6 \mathrm{a}$, the $1_{1}$ level of the benzene dimer decays exponentially and the decay

(a) Benzene dimer

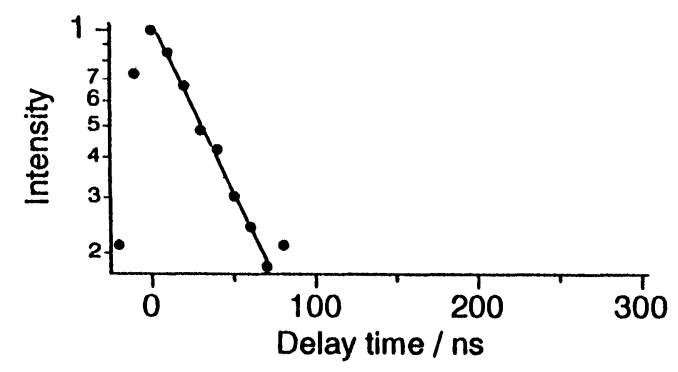

(b) Benzene - Ar

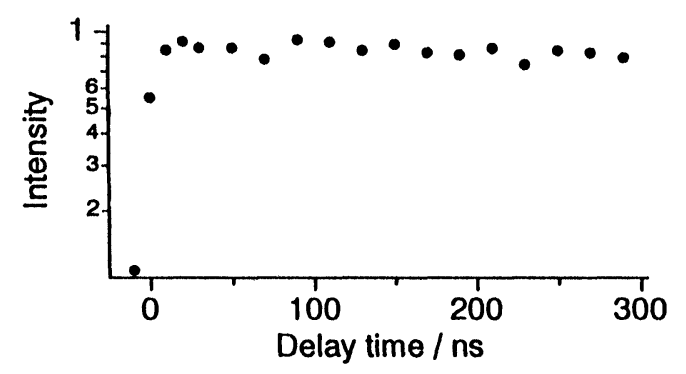

(c) Benzene - $\mathrm{N}_{2}$

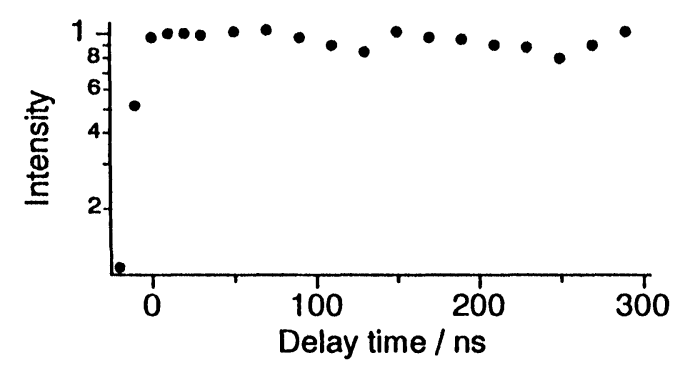

Figure 6 Decay profiles of the $1_{1}$ levels of (a) benzene dimer, (b) benzene-Ar $(1: 1)$ complex and (c) benzene- $\mathrm{N}_{2}(1: 1)$ complex. 
rate constant was obtained to be $2.4( \pm 0.3) \times 10^{7} \mathrm{~s}^{-1}$, which is almost the same as was reported previously. On the other hand, the intensities of the ionization signal from the $1_{1}$ levels of the benzene-Ar and benzene- $\mathrm{N}_{2}$ complexes did not show decay for $300 \mathrm{~ns}$, which was the maximum delay time under the present condition. Their decay rate constants should be much smaller than $1 \times 10^{6} \mathrm{~s}^{-1}$.

Two relaxation processes are possible for the benzene complexes after exciting to the $1_{1}$ level by stimulated Raman pumping. One is intramolecular vibrational redistribution (IVR) and the other is vibrational predissociation (VP). The former process will be followed by the dissociation of the complex. The binding energies of the benzene dimer, benzene-Ar and benzene- $\mathrm{N}_{2}$ complexes have been reported to be $\sim 500 \mathrm{~cm}^{-1}$, and the vibrational energy of mode $1\left(993 \mathrm{~cm}^{-1}\right)$ is large enough to break the van der Waals bond. In both processes, the rate of the primary process is expressed by the Golden rule,

$$
\mathrm{k}=\frac{2 \pi}{\hbar} \sum_{\mathrm{f}}|\langle\mathrm{i}|\mathrm{V}| \mathrm{f}\rangle|^{2} \cdot \delta[\mathrm{E}(\mathrm{i})-\mathrm{E}(\mathrm{f})] .
$$

Here, $\mid i>$ and $|f\rangle$ are the initial state and the manifold of the final states coupled by $\mathrm{V}$. V is the vibrational anharmonicity of the mode 1 coming mainly from coupling with van der Waals modes. Since the van der Waals mode is cold and ring mode is excited in the initial state, the initial state is expressed by $|\mathrm{i}\rangle=\left|\mathrm{v}^{\prime \prime}{ }_{\text {ring }}, 0\right\rangle$. The final state is $|f\rangle=\left|v_{\text {ring }}^{\prime}, v_{\text {van }}^{\prime}\right\rangle$. $v_{\text {van }}^{\prime}$ is discrete vibrational levels for IVR, while it is continuum state for V.P. Finally, we obtain

$$
\mathrm{k}=\frac{2 \pi}{\hbar} \mathrm{K}_{\mathrm{r}_{\text {ring }}^{\prime \prime}}, 0|\mathrm{v}| \mathrm{v}_{\text {ring, }}^{\prime}, \mathrm{v}_{\text {van }}^{\prime}>\left.\right|^{2} \cdot \rho(\mathrm{E}) \text {. }
$$

Here $\rho$ is the density of the final states. We will discuss the observed decay rates based on this equation.

There are two interesting points in the observed results. First is the great difference in the decay rate constant between the benzene dimer and the other complexes, that is the vibrational relaxation of the dimer is much faster than those of benzene-Ar and benzene- $\mathrm{N}_{2}$ complexes. Since the vibrational frequency shift is less than $1 \mathrm{~cm}^{-1}$ for all the complexes as mentioned previously, the matrix element in eq. (12) is not so different among the complexes studied. Therefore, the great difference may be attributed to the difference in the density of states of the final state. If the relaxation is due to the vibrational predissociation, the density of the continuum levels is expressed as ${ }^{69}$

$$
\rho(\mathrm{E})=\rho_{\mathrm{T}}\left(\mathrm{E}_{\mathrm{T}}\right)=\sqrt{2} \mu^{\frac{3}{2}} \pi^{2} \mathrm{~h}^{3} \mathrm{E}_{\mathrm{T}}^{\frac{1}{2}} .
$$

Here $\mu$ is the reduced mass for the intermolecular stretching mode. $\mathrm{E}_{\mathrm{T}}$ is relative translational energy and is given by

$$
\mathrm{E}_{\mathrm{T}}=\mathrm{E}-\text { B.E. }-\mathrm{E}_{\mathrm{INT}} \text {. }
$$

Here, B.E. is the binding energy of the complex and $\mathrm{E}_{\mathrm{INT}}$ is the sum of the internal energies of the fragments. As described previously, the binding energy is not so 
different among the complexes. So, the difference in $\rho(\mathrm{E})$ is attributed to the difference in the term of the reduced mass $\mu^{3 / 2}$. The ratios of $\mu^{3 / 2}$ of benzene- $\mathrm{N}_{2}$ and benzene-Ar complexes to that of the benzene dimer are 0.38 and 0.56 , respectively. Therefore, they differ only by a factor of 2 , which is too small to explain the observed difference in the relaxation rate constants.

On the other hand, if the relaxation is due to the IVR, the density of states of the final states will be greatly different among the complexes. First, the benzene dimer has six intermolecular vibrational modes which is larger than five for benzene- $\mathrm{N}_{2}$ or three for benzene-Ar. Beside the number of the intermolecular mode, we see a great difference in the frequency of the mode. For example, we shall consider the rotational motion of the benzene molecule on stem site around the $\mathrm{C}_{6}$ axis of the benzene on the top site passing through the in-plane $\mathrm{C}_{2}$ axis of the stem site benzene. The benzene- $\mathrm{N}_{2}$ complex has also a similar vibrational mode. It is readily seen that the vibrational frequency of this rotational mode is much smaller in benzene dimer than that in benzene- $\mathrm{N}_{2}$ as shown by the estimated rotational constants of $\mathrm{B}=$ $2.11 \mathrm{~cm}^{-1}$ and $0.284 \mathrm{~cm}^{-1}$, respectively, for benzene- $\mathrm{N}_{2}$ and $\mathrm{T}$ shaped-dimer. The benzene-Ar complex does not have this mode. The frequencies of other intermolecular modes are also expected to be smaller in the benzene dimer considering the larger mass of benzene molecule. From the differences in the number of the modes and their frequencies between the benzene dimer and the benzene- $\mathrm{N}_{2}$ (or benzene-Ar), the density of state of the benzene dimer is expected to be about ten times larger than those of the other complexes at the energy level of $1000 \mathrm{~cm}^{-1}$. Another important point is that since the dimer contains two benzene molecules, a near resonant energy transfer will occur effectively in the dimer. This energy transfer together with the large state density may be the reason why the IVR of the dimer is much faster than the other complexes.

Finally, we shall briefly discuss the difference in the relaxation rate between the $\mathrm{S}_{0}$ and $\mathrm{S}_{1}$ states. In the present experiment, the vibrational relaxation rates of the mode 1 level of the benzene-Ar and benzene- $\mathrm{N}_{2}$ complexes in $\mathrm{S}_{0}$ were estimated to be much smaller than $1 \times 10^{6} \mathrm{~s}^{-1}$. On the other hand, the reported relaxation rates of the complexes in the $S_{1}$ state is equal to or faster than the fluorescence decay rate $\left(10^{8} \sim 10^{7} \mathrm{~s}^{-1}\right)$. In the case of the benzene-Ar complex, the IVR rate constant in $S_{1}$ is reported to be $7 \times 10^{7} \mathrm{~s}^{-1}$ for the $1^{1} 6^{1}$ level $\left(1,444 \mathrm{~cm}^{-1}\right)$ by Stephenson et al. ${ }^{27}$ Therefore, the vibrational relaxation rate of benzene-Ar complex in $S_{1}$ is more than two orders of magnitude larger than that in $S_{0}$. This may be also true in the benzene- $\mathrm{N}_{2}$ complex. The result indicates that the IVR and VP processes are greatly affected by the electronic state. Recently, Weber et al. also found slower vibrational relaxation in $S_{0}$ than in $S_{1}$ in s-tetrazine-rare gas complexes..$^{70}$ They reported that the lifetimes of the vibrational levels of the complexes due to IVR/VP are about $15 \mathrm{~ns}$ in $S_{0}$, while those in $S_{1}$ are reported to be $\sim 2$ ns by the group of Levy. ${ }^{25}$ Therefore, the present result shows a similar behavior. The lifetime of the complex due to IVR/VP is $14 \mathrm{~ns}$ in $\mathrm{S}_{1}$, while it is longer than $1 \mu \mathrm{s}$ in $\mathrm{S}_{0}$.

As was described previously, the relaxation rate is expressed by eq. (12) for any electronic states. Therefore, the difference of the rate should be attributed either to the difference in the density of final states and/or to the difference in the coupling 
matrix element. Since the intermolecular vibrational modes were not observed in the $S_{1}-S_{0}$ spectrum, the van der Waals potentials in $S_{1}$ and $S_{0}$ will be very similar. Therefore, the density of states will be similar between the two electronic states and the difference in the rate constant may be attributed to the difference in the matrix element in eq. (12). Weber et al. pointed out that the mode 16a showing a large frequency change by the complex formation is playing an important role in the fast relaxation in $S_{1}$ of the s-tetrazine-rare gas complexes. ${ }^{70}$ A similar large vibrational frequency change was also observed for the mode 16 of benzene in the $S_{1}-S_{0}$ spectra of benzene-rare gas complexes. ${ }^{27}$ Such a great difference in the IVR/VP rate constant between $S_{1}$ and $S_{0}$ seems to be quite general for other aromatic van der Waals complexes, being worthy for more intensive investigation.

\section{Acknowledgments}

The authors thank N. Mikami, M. Fujii and Y. Fujimura for their helpful discussions.

\section{References}

1. D. H. Levy. Adv. Chem. Phys., 47, 3742 (1981).

2. A. Amirav and U. Even and J. Jortner. J. Chem. Phys., 75, 2489 (1981).

3. A. Amirav and J. Jortner. J. Chem. Phys., 85, 19 (1981).

4. S. Leutwyler. J. Chem. Phys., 81, 5480 (1984).

5. S. Leutwyler and J. Jortner. J. Phys. Chem., 91, 5558 (1987).

6. T. Troxler, R. Knochenmuss and S. Leutwyler. Chem. Phys. Lett., 159, 554 (1989).

7. S. Leutwyler and J. Bosiger. Chem. Rev., 90, 489 (1990).

8. T. Troxler and S. Leutwyler. J. Chem. Phys., 95, 4010 (1991).

9. J. C. Kettley, J. W. Oram, T. F. Palmer, J. P. Simons and A. T. Amos. Chem. Phys. Lett., 140, 286 (1987).

10. M. Y. Hahn and R. L. Whetten. Phys. Rev. Lett., 61, 1190 (1988).

11. X. Li, M. Y. Hahn, M. S. Al-Shall, R. L. Whetten. J. Phys. Chem., 95, 8524 (1991).

12. E. R. Bernstein. J. Phys. Chem., 96, 10105 (1992).

13. M. Shmidt, M. Mons and J. Le Calve. Chem. Phys. Lett., 177, 371 (1991).

14. M. Schmidt, M. Mons and J. Le Calve. Ber. Bunsenges. Chem., 96,1284 (1992).

15. M. Mons, J. Le Calve, F. Piuzzi and I. Dimicoli. J. Chem. Phys., 92, 2155 (1992).

16. C. A. Haynam, D. V. Brumbaugh and D. H. Levy. J. Chem. Phys., 79, 1581 (1983).

17. C. A. Haynam, D. V. Brumbaugh and D. H. Levy. J. Chem. Phys., 80, 2256 (1984).

18. S. M. Beck, M. G. Liverman, D. L. Monts and R. E. Smalley. J. Chem. Phys., 70, 232 (1979).

19. Y. Sugahara, N. Mikami and M. Ito. J. Phys. Chem., 90, 5619 (1986).

20. D.H. Levy. In Structure and Dynamics of Weakly Bound Molecular Complexes; A. Weber Ed. NATO ASI series C, Vol. 211, p. 231 (1986).

21. W. M. van Herpen and W. L. Meerts. Chem. Phys. Lett., 147, 7 (1988).

22. Th. Weber, A. von Bargen, E. Riedle, and H. J. Neusser. J. Chem. Phys., 92, 90 (1990).

23. Th. Weber, A. M. Smith, E. Riedle, H. J. Neusser and E. W. Schlag. Chem. Phys. Lett., 175, 79 (1990).

24. P. R. R. Langridge-Smith, E. Carrasquillo and D. H. Levy. J. Chem. Phys., 74, 6513 (1981).

25. D. V. Brumbaugh, J. E. Kenny and D. H. Levy. J. Chem. Phys., 78, 3415 (1983).

26. N. Halbersadt and B. Soep. J. Chem. Phys., 80, 2340 (1984).

27. T. A. Stephenson and S. A. Rice. J. Chem. Phys., 81, 1083 (1984).

28. H. Abe, Y. Ohyanagi, M. Ichijo, N. Mikami and M. Ito. J. Phys. Chem., 89, 3512 (1985).

29. K. W. Butz, D. L. Catlett Jr., G. E. Ewing, D. Krajnovich and C. S. Parmenter. J. Phys, Chem., 90, 3533 (1986).

30. T. Kobayashi and O. Kajimoto. J. Chem. Phys., 86, 1118 (1987). 
31. H. Saigusa and M. Itoh. J. Chem. Phys., 86, 2588 (1987).

32. A. L. Motyka, S. A. Wittmeyer, R. J. Babbitt and M. R. Topp. J. Chem. Phys., 89, 4586 (1988).

33. H. K. O, C. S. Parmenter and M. C. Su. Ber. Bunsenges, Physik. Chem., 92, 253 (1988).

34. T. S. Zwier. AIP conf. Proc., 191, 692 (1989).

35. D. H. Semmens, J. S. Baskin and A. H. Zewail. J. Chem. Phys., 92, 3359 (1990).

36. A. Kazisha and M. R. Topp. Chem. Phys. Lett., 180, 423 (1991).

37. A. Heikal, L. Banares, D. H. Semmens and A. H. Zewail. Chem. Phys., 156, 231 (1991).

38. J. C. Alfano, S. J. Martinez and D. H. Levy. J. Chem. Phys., 96, 2522 (1992).

39. J. Knee and P. M. Johnson. J. Chem. Phys., 80, 13 (1984).

40. J. Knee and P. M. Johnson. J. Phys. Chem., 89, 3512 (1985).

41. A. Sur and P. M. Johnson. J. Chem. Phys., 84, 1206 (1986).

42. J. F. Knee, L. R. Khundkar and A. H. Zewail. J. Chem. Phys., 87, 115 (1987).

43. B. A. Jacobson, S. Humphrey and S. A. Rice. J. Chem. Phys., 89, 5624 (1988).

44. R. J. Lipert and S. D. Colson. J. Phys. Chem., 94, 2358 (1990).

45. P. Escherick and A. Owyoung. Chem. Phys. Lett., 103, 235 (1983).

46. P. Escherick, A. Owyoung and J. Pliva. J. Chem. Phys., 83, 3311 (1985).

47. J. Pliva, P. Escherick and A. Owyoung. J. Mol. Spectrosc., 125, 393 (1987).

48. G. V. Hartland, B. F. Henson, V. A. Venturo and P. M. Felker. J. Phys. Chem., 96, 1164 (1992).

49. B. F. Henson, G. V. Hartland, V. A. Venturo and P. M. Felker. J. Chem. Phys., 91, 2751 (1989).

50. B. F. Henson, G. V. Hartland, V. A. Venturo, R. A. Hertz and P. M. Felker. Chem. Phys. Lett., 176, 91 (1991).

51. V. A. Venturo, P. M. Maxton and P. M. Felker. J. Phys. Chem., 96, 5234 (1992).

52. V. A. Venturo, P. M. Maxton and P. M. Felker. Chem. Phys. Lett., 198, 628 (1992).

53. B. F. Henson, G. V. Hartland, V. A. Venturo and P. M. Felker. J. Chem. Phys., 97, 2189 (1992).

54. V. A. Venturo, P. M. Maxton, B. F. Henson and P. M. Felker. J. Chem. Phys., 96, 7855 (1992).

55. T. Ebata, M. Hamakado, S. Moriyama, Y. Morioka and M. Ito. Chem. Phys. Lett., 199, 33 (1992).

56. S. Hyodo and T. Fujiyama. Bull. Chem. Soc. Jpn., 53, 2456 (1980).

57. W. Scherzer, O. Kratzschmar, H. L. Selzle and E. W. Schlag, Z. Naturforsch. 47a, 1248 (1992).

58. B. W. van de Waal. Chem. Phys. Lett., 123, 69 (1986).

59. M. L. Allinger and L. Jenn-Huei. J. Comput. Chem., 8, 1146 (1987).

60. P. Carsky, H. L. Selzle and E. W. Schlag. Chem. Phys., 125, 165 (1988).

61. P. Hobza, H. L. Selzle and E. W. Schlag. J. Chem. Phys., 98, 5893 (1990).

62. P. Hobza and R. Zahradnik. Ber. Bunsenges. Phys. Chem., 96, 1294 (1992).

63. For example, K. Morokuma. J. Chem. Phys., 55, 1236 (1971); K. Kitaura and K. Morokuma. Int. J. Quantum Chem., 10, 325 (1976); S. Yamabe and K. Morokuma. J. Am. Chem. Soc., 97, 4458 (1975).

64. E. B. Wilson, J. C. Decius and P. C. Cross, "Molecular Vibrations", McGraw-Hill, 1955.

65. A. Kiermeiner, B. Ernstberger, H. J. Neusser and E. W. Schlag. J. Phys. Chem., 92, 3785 (1988).

66. H. Krause, B. Ernstberger and H. J. neusser. Chem. Phys. Lett. 184, 411 (1991).

67. P. Hobza, O. Bludsky, H. L. Selzle and E. W. Schlag. J. Chem. Phys., 97, 335 (1992).

68. R. Nowak, J. A. Menapace and E. R. Bernstein. J. Chem. Phys., 89, 1309 (1988).

69. A. Ben-Shaul, Y. Haas, K. L. Kompa and R. D. Levine, "Lasers and Chemical Change" Springer Series in Chemical Physics 10, 1981, Springer-Verlag, Berlin, Heidelberg, New York.

70. P. M. Weber and S. A. Rice. J. Chem. Phys., 88, 6120 (1988).

71. Landolt-Börnstein, “Atom u Mol. Phys. Teil 3, Moleculen II"' Springer-Verlag, 1951.

72. L. Pauling, "The Nature of Chemical Bond", Cornell Univ. Press, 1960.

73. A. H. Narten. J. Chem. Phys., 67, 2102 (1977).

74. G. Karlström, P. Linse, A. Wallqvist and B. Jönsson. J. Am. Chem. Soc., 105, 3777 (1983). 